\title{
Über die normativen Implikationen des Ordoliberalismus für die moderne Wirtschaftsethik ${ }^{*}$
}

\author{
RAINER KLUMP UND MANUEL WÖRSDÖRFER ${ }^{* *}$
}

\section{The Ethical-Normative Implications of German Ordoliberalism}

Eucken's writings are rich in content not only from an (constitutional) economic perspective but also from an economic and business ethics perspective - an often neglected perspective of the reception of German Ordoliberalism in general and the Freiburg School of Economics in particular. The paper gives a first insight into the ethical-normative thinking of Eucken, as one of the main representatives of Ordoliberalism, and explains Eucken's conception of Social Market Economy as a functioning and humane order as well as Eucken's Kantian understanding of freedom. To conclude the essay tries to classify Eucken's Ordoliberalism as a part of the spectrum of modern economic and business ethics.

Keywords: Ordoliberalism, Freiburg School of Economics, Walter Eucken, Liberty, Kantian Autonomy

\section{Einleitung}

Die moderne deutschsprachige Wirtschaftsethik wurde nachhaltig geprägt durch die Arbeiten von Karl Homann und Peter Ulrich sowie die jeweils in ihrer Tradition stehenden Schüler. ${ }^{1}$ Homann vertritt dabei einen moralökonomischen Ansatz, der auf Buchanan und der Neuen Institutionenökonomik (NIÖ) aufbaut; Ulrichs Integrative Wirtschaftsethik hingegen ist der Kantischen Philosophie und vor allem der Diskursethik verpflichtet. Bemerkenswert ist, dass beide dichotomen und partiell inkommensurablen Konzepte sich explizit mit dem Ordoliberalismus, einem Wegbereiter der Sozialen Marktwirtschaft, auseinander setzen. Während die zum Kern des Ordolibera-

\footnotetext{
* Beitrag eingereicht am 3.07.2009; nach doppelt verdecktem Gutachterverfahren überarbeitete Fassung angenommen am 15.09.2009. Die Verfasser danken den anonymen Gutachtern und besonders Dr. Olaf Schumann, Goethe-Universität Frankfurt am Main, für ihre hilfreichen Anmerkungen und ihre konstruktive Kritik.

** Prof. Dr. Rainer Klump, Lehrstuhl für VWL, insb. Wirtschaftliche Entwicklung und Integration, Goethe-Universität, Grüneburgplatz 1, Postfach 1119 32, 60629 Frankfurt am Main, Tel.: 069798-34787, Fax: 069-798-35015, E-Mail: klump@wiwi.uni-frankfurt.de, Forschungsschwerpunkte: Wachstum und Entwicklung, Europäische Integration, Normative Grundlagen der Sozialen Marktwirtschaft, Wirtschaftspolitik; Manuel Wörsdörfer, M. A., Wissenschaftlicher Mitarbeiter des Exzellenzclusters „Die Herausbildung normativer Ordnungen“, Goethe-Universität, Frankfurt am Main, Tel.: 069-798-34778, E-Mail: woersdoerfer@wiwi.uni-frankfurt.de, Forschungsschwerpunkte: Normative Grundlagen des Ordoliberalismus, Wirtschafts- und Unternehmensethik.

1 Es sei an dieser Stelle darauf hingewiesen, dass sich die deutschsprachige Wirtschafts- und Unternehmensethik natürlich nicht auf diese beiden Ansätze reduzieren lässt. So existieren weitere bedeutende Ansätze sowie nennenswerte wirtschaftsethische Forschung, die ansatzunabhängig argumentiert.
} 
lismus zählende Freiburger Schule der Nationalökonomie von Vertretern der NIÖ (vgl. Homann/Lütge 2004/2005; Pies 2002; Suchanek 2001/2007; Vanberg 2000) z. T. selektiv rezipiert respektive einseitig interpretiert wird, erhebt Ulrich schwere Vorwürfe gegen einige der Vertreter des Ordoliberalismus: So wirft er ihnen vor, einem „,Rückfall" in altes neoklassisches Denken“ Vorschub zu leisten, eine „systematisch ungenügende und unzeitgemäße politisch-philosophische Fundierung ihrer ordnungspolitischen Konzeption" zu besitzen und letztlich bloß eine korrektive, nicht jedoch eine integrative Wirtschaftsethik zu sein (Ulrich 1997/2008: 384f. und 389; Nawroth 1965: 23ff.; Ptak 2007).

Beide Arten des Umgangs mit dem Ordoliberalismus bedürfen einer Kritik. Dabei ist es hilfreich, auf die Originalbeiträge zurückzugehen und zwischen den einzelnen Vertretern zu differenzieren. Im Mittelpunkt des vorliegenden Beitrags steht Walter Eucken, Hauptvertreter der Freiburger Schule und des Ordoliberalismus im engeren Sinne. ${ }^{2}$ Euckens Schriften, die zu den Klassikern des ökonomischen Denkens im deutschsprachigen Raum zählen, bieten nicht nur für die NIÖ eine Vielzahl von Anknüpfungspunkten (vgl. Oberender/Christl 2000; Erlei/Leschke/Sauerland 1999/2007); auch aus wirtschaftsethischer Perspektive, ein Blickwinkel, der bei der Rezeption des Freiburger Nationalökonomen häufig vernachlässigt wird, ${ }^{3}$ sind sie ein reichhaltiger wirtschaftsordnungspolitischer Fundus, der gerade mit Blick auf die gegenwärtige Finanzmarkt- und Weltwirtschaftskrise kaum an Aktualität verloren hat. ${ }^{4}$

Der vorliegende Aufsatz beabsichtigt, Einblicke in das ethisch-normative Denken von Eucken zu geben, und versucht dieses in seinen elementaren Grundzügen nachzuzeichnen. Dabei wird es neben der Bestimmung des Staates als ordnender Faktor insbesondere um Euckens an Kant angelehntes Menschenbild und Freiheitsverständnis gehen sowie um eine Einordnung Euckens in die moderne Wirtschafts- und Unternehmensethik. Es wird sich zeigen, dass sich Euckens ordoliberale Konzeption im Spannungsfeld von Integrativer Wirtschaftsethik und Moralökonomik bewegt, dabei aber eine eigenständige Position einnimmt. ${ }^{5}$

2 Die Freiburger Schule war eine interdisziplinäre Lehr- und Forschungsgemeinschaft in den 1930er und 40er Jahren, bestehend aus Juristen und Ökonomen, an der Universität Freiburg. Mitglieder der Freiburger Schule bzw. des Ordoliberalismus i. e. S. waren, neben Eucken, Böhm und Großmann-Doerth. Zu den Vertretern des Ordoliberalismus i. w. S. zählen Röpke, Rüstow, MüllerArmack, Erhard u. a. (vgl. Goldschmidt/Wohlgemuth (2008: 1)).

3 Eine bedeutende Ausnahme ist Goldschmidt: vgl. Goldschmidt (2002) und (2003) sowie Blümle/Goldschmidt (2000). Erwähnenswert ist weiterhin Schlecht (1990); allerdings ist hier die wirtschaftsethische Perspektive nur sehr rudimentär ausgebildet.

$4 \quad \mathrm{Zu}$ nennen sind hier die Frage der staatlichen Regulierung und Kontrolle von Finanzmarktprodukten, die Frage der Transparenz in den einzelnen Marktsegmenten, die Frage der Haftung der Ratingagenturen und der Managerhaftung/-verantwortung sowie die Frage der (zunehmend oligopolistischen) Machtkonzentration im Finanzdienstleistungssektor.

$5 \quad$ An dieser Stelle sei darauf verwiesen, dass es nicht das primäre Ziel des vorliegenden Aufsatzes ist, die Integrative Wirtschaftsethik und die Moralökonomik kritisch zu bewerten. Vielmehr geht es darum, Überschneidungen und Divergenzen der beiden Ansätze mit demjenigen von Eucken aufzuzeigen und somit den Weg zu ebnen für eine differenzierte, ganzheitliche und weniger vorurteilsbehaftete Herangehensweise an die Texte Euckens. Schließlich geht es uns auch um die Herausarbeitung des (vermittelnden) wirtschaftsethischen Potenzials der Lehre Euckens. 
Unsere Argumentation ist wie folgt gegliedert: Der nachfolgende Abschnitt beschäftigt sich mit dem Euckenschen Anforderungsprofil an eine Soziale Marktwirtschaft. Demnach hat eine sozialmarktwirtschaftliche Wirtschaftsordnung sowohl das Kriterium der Funktionsfähigkeit als auch dasjenige der Menschenwürdigkeit zu erfüllen (Kapitel 2). Im dritten Abschnitt wird Euckens an Kant angelehntes Freiheits- und Autonomieverständnis erläutert (Kapitel 3). Hieran anschließend thematisieren wir Euckens Staatskonzeption, seine Vorstellungen von den Grenzen der Staatstätigkeit und die ordoliberalen Implikationen für die Relation Markt vs. Staat (Kapitel 4). Der fünfte Abschnitt schließlich vergleicht Euckens Ordoliberalismus mit der Moralökonomik Homanns und der Integrativen Wirtschaftsethik Ulrichs (Kapitel 5). Der Aufsatz endet mit einem Resümee und einem Ausblick (Kapitel 6).

\section{Soziale Marktwirtschaft als funktionsfähige und menschenwürdige Ordnung}

Eine bedeutsame Konstante im Werk Euckens ist das vielfach vorgetragene duale $A n$ forderungsprofil an eine mark.twirtschaftliche und sozial verpflichtete Wettbewerbsordnung: Einerseits muss eine derartige Wirtschaftsordnung in Abgrenzung zur Zentralverwaltungswirtschaft, zum Laissez-faire-Kapitalismus und zum Zeitalter der „Wirtschaftspolitik der Experimente“ (Eucken 1952/2004: 55ff.) effizient und produktiv funktionieren. Festgemacht wird die ökonomische Leistungsfähigkeit an einem möglichst effizienten und nachbaltigen Umgang mit Knappheit und an einer möglichst umfassenden Versorgung der Bevölkerung mit (lebensnotwendigen) Wirtschaftsgütern. Die bestmögliche Versorgung ist laut Eucken in einem marktwirtschaftlichen System garantiert (vgl. bereits Eucken 1926 und 1930). Allerdings ist die Funktionsfähigkeit einer Wirtschaftsordnung kein Selbstzweck: Sie liefert vielmehr das Fundament für ein menschenwürdiges, „lebensdienliches“ und „vitalpolitisches“6 Leben und das bedeutet bei Eucken ein autonomes, eigenverantwortliches Leben in Freiheit. ${ }^{7}$ Indem die marktwirtschaftliche Ordnung für eine ausreichende Befriedigung der materiellen Grundbedürfnisse sorgt, schafft sie gleichsam die Voraussetzung für die Realisierung einer darüber hinaus gehenden, immateriellen Entfaltung der intellektuellen Persönlichkeit und für die Wahrung respektive Wahrnehmung fundamentaler Menschenrechte. Das außer-, nicht jedoch meta-ökonomische Kriterium (d. i. die Menschenwürdigkeit der Wirtschafts- und Rechtsordnung) hat dabei auch Rückwirkungen auf das Kriterium der Gleichgewichtigkeit der Wettbewerbsordnung - zu erwähnen ist die die Funktionsfähigkeit ermöglichende bzw. fördernde gesellschaftliche Kohäsion und Stabilität infolge einer tausch- und verteilungsgerechten Ordnung. Insofern ist Eucken zuzustimmen, wenn er von einer Interdependenz und Komplementarität der beiden staatlich zu implementierenden Kriterien einer Sozialen Marktwirtschaft spricht. Eine Rangfolge der Kriterien existiert ebenso

6 Beide: Ulrich (1997/2008); Ulrich (a. a. O.: 378) spricht hier auch von der instrumentellen „Zweckdienlichkeit der Marktwirtschaft“. Der von Ulrich so häufig zitierte Begriff der Vitalpolitik ist den Werken Alexander Rüstows entlehnt (vgl. u. a. Rüstow (1955: 74)).

$7 \quad$ Freiheit ist bei Eucken stets an Gerechtigkeit und Humanität gekoppelt; gelegentlich ersetzt Eucken (1952/2004: 332) Menschenwürdigkeit durch (die Synonyme) Freiheit und Gerechtigkeit. 
wenig wie eine Über- oder Unterordnung; beide Kriterien sind gleichrangig und ebenbürtig. Es gibt folglich kein Primat der Ethik oder der Ökonomie! ${ }^{8}$

Eucken schreibt: „Die Aufgabe bleibt immer die gleiche: die Herstellung einer funktionsfähigen und gerechten (d. i. menschenwürdigen) Ordnung. (...) Die Funktionsfähigkeit ist eine Frage des Gleichgewichts. Nicht weniger aber ist es (...) die Gerechtigkeit. Dem Gleichgewicht kommt also mehr als eine bloß ökonomisch-technische Bedeutung zu. "

Eucken verweist mit seinem ökonomisch-philosophischen Kriteriendualismus auf eine weitere ordoliberale Besonderheit: Wirtschaftspolitik, vertreten durch das erste Kriterium, und Sozialpolitik, repräsentiert durch das zweite angeführte Kriterium, sind und dies ist entscheidend - auf der Ebene der (Wirtschafts-)Verfassungspolitik miteinander verzahnt. Sozialpolitik ist primär (Wirtschafts-)Ordnungspolitik und sie ist nicht gegen oder für den Markt, sondern nur mit dem Markt operationalisierbar ${ }^{10}$ - insofern ist von einer grundlegenden Reziprozität der beiden Kriterien auszugehen. Schließlich verweist die enge Verzahnung der beiden Kriterien einer Wirtschaftsordnung auf die von Eucken so häufig herausgestellte Interdependenz, der Ordnungen (d. i. Interdependenz der politisch-juridischen, soziokulturellen und ökonomischen Ordnung). Diese Interdependenz und das hiermit verbundene Denken in ordnungsspezifischen Gesamtzusammenhängen gilt es bei den jeweiligen (wirtschafts- und sozial-)politischen Entscheidungen zu berücksichtigen. ${ }^{11}$

Überaus wichtig ist allerdings, dass die Kriterien der Humanität und Gerechtigkeit nur dann realisiert werden können, wenn die Marktverfassung, oder wie Eucken sich ausdrückt: die Rahmenordnung der Verkehrswirtschaft, von staatlicher Seite aus mark.tkonform reguliert wird. Der Staat hat mit seiner Ordnungspolitik dafür Sorge zu tragen, dass wirtschaftliche Transaktionen in einem System der vollständigen Konkurrenz ablaufen, und er hat dafür zu sorgen, dass der natürlichen Tendenz zur Vermachtung ${ }^{12}$ in der politökonomischen Sphäre frühzeitig und präventiv begegnet wird.

Zusammengefasst werden Euckens Vorstellungen einer adäquaten „Leistungswettbewerbs"13_Ordnung in den beiden Grundsätzen der Wirtschaftspolitik sowie in den konstituierenden und regulierenden Prinzipien. Während der erste Grundsatz der Wirtschaftpolitik die Entmachtung politökonomischer ,pressure groups“ verlangt, geht es dem zweiten Grundsatz um die Verpflichtung des Staates auf die Ordnungspolitik und die Entsagung

$8 \quad$ Vgl. Eucken (1938: 81). Eucken wendet sich hier explizit gegen eine Verabsolutierung der Ökonomie.

$9 \quad$ Eucken (1952/2004: 166); vgl. ebenfalls (a. a. O.: 14 und 369). Die Euckenschen Kriterien Funktionsfähigkeit und Menschenwürdigkeit beziehen sich nicht nur auf die Wirtschaftsordnung, sondern auch auf die Gesellschafts-, Rechts- und Staatsordnung (vgl. Eucken (1950/1965: 239f.)).

$10 \quad$ Vgl. Blümle (2007/2008: 299).

11 Vgl. zur Diskussion der Interdependenz der Ordnungen sowie zum Denken in Ordnungen: Eucken (1950/1965: 50ff. und 62) sowie Eucken (1940) und (1952/2004: 13ff., 19ff. und 183).

12 Eucken (1952/2004: 31) spricht diesbezüglich auch von einem natürlichen „Hang zur Monopolbildung".

13 Vgl. u. a. Eucken (1952/2004: 247). Eucken grenzt den Leistungswettbewerb gezielt vom sog. Behinderungswettbewerb ab (vgl. a. a. O.: 267 und 297). 
jeglicher Art von Prozesspolitik: Die Hauptaufgabe des Staates ist in HayekEuckenscher Terminologie in der Gestaltung allgemeiner abstrakter Spielregeln (nomos) zu sehen und nicht in interventionistischen Einzelfallmaßnahmen (thesis) respektive dem Eingreifen in die ökonomischen Spielzüge der Wirtschaftssubjekte, da dieses interessengruppenbasiert, willkürlich, ungerecht und - was am bedeutsamsten ist wohlfahrts- und freibeitsmindernd wäre. Die Lenkung und Planung des Wirtschaftsprozesses übersteigt aufgrund von Anreiz- und Informationsrestriktionen (,Anmaßung von Wissen“ (vgl. Hayek 1975)) die Möglichkeiten und Fähigkeiten des Staates; darüber hinaus erhöht sie empfindlich den Einflussspielraum von Rent-seekingAktivitäten. Die Fokussierung auf die Gestaltung der Ordnungsformen sowie der hiermit einhergehende „Verzicht auf unmittelbare Lenkung durch laufende Eingriffe“ (Eucken 1952/2004: 337) ist gemäß Eucken der einzig gangbare Weg. Die Ordnungsformen, in denen gewirtschaftet wird, werden maßgeblich bestimmt durch die sog. konstituierenden und regulierenden Prinzipien.

An dieser Stelle sei nur kurz auf die in diesem Zusammenhang relevantesten Postulate eingegangen: In Anlehnung an Böhm und Miksch ist Wettbewerb als staatliche Veranstaltung und als rechtschöpferischer Akt aufzufassen (vgl. Böhm 1937; Miksch 1937/1947). Eine wichtige Voraussetzung zur Realisierung der Marktform der vollständigen $^{14}$ Konkurrenz ist ein unabhängiger, neutraler und leistungsfähiger Rechtsstaat, der in der Lage ist, Partikularinteressen abzuwehren, Marktmacht und marktbeherrschenden Positionen präventiv zu begegnen und eine aktive, konstante und prinzipiengeleitete Wettbewerbspolitik zu betreiben. Letzteres bezieht sich sowohl auf die Etablierung offener Märkte (d. i. Verhinderung von Marktzutrittsbeschränkungen und Vermeidung von Konzentrationstendenzen) als auch auf eine unabhängige und konsequente Monopolkontrolle (erstes regulierendes Prinzip). Neben der auch gesellschaftsund stabilitätspolitisch so bedeutsamen Preisniveaustabilität tritt Eucken für originär liberale Positionen wie Privateigentum und Vertragsfreiheit ein. Diese werden jedoch keineswegs verabsolutiert: Jedes der genannten Prinzipien wird gewissen Bedingungen unterworfen und somit relativiert. Privateigentum an den Produktionsmitteln bedarf einer unbedingten Kontrolle durch das marktwirtschaftliche Konkurrenzsystem und das Haftungs- bzw. Verantwortungsprinzip. Die Vertragsfreiheit ist ebenfalls nur eine relative, da Wettbewerb ein- bzw. beschränkende Verträge nicht gestattet sind. Freiheit findet ganz allgemein gesprochen immer dort ihre Grenze, wo die Freiheit und Rechte Anderer bedroht sind; die Freiheitssphäre Dritter gilt es unbedingt zu respektieren (vgl. Kant 1977a: 336ff.; 1977b: 145f.).

Schließlich erweitert Eucken die konstituierenden Prinzipien um vier regulierende, ${ }^{15}$ die eine (moderate) Korrektur der Markt- und Verteilungsergebnisse insbesondere beim Vorliegen von Marktversagen ermöglichen sollen; die Wettbewerbsordnung bzw. Soziale Marktwirtschaft ist - ähnlich wie die Demokratie - nur die „relativ beste Lösung“" (Eucken 1952/2004: 300); auch sie enthält gewisse Mängel, Risiken und Gefah-

Eucken grenzt bewusst sein Konzept der Marktform der vollständigen Konkurrenz von demjenigen der Neoklassik (d. i. vollkommene Konkurrenz) ab (vgl. u. a. Eucken (1952/2004: 279)).

Das erste der vier regulierenden Prinzipien wurde aus inhaltlichen Gründen bereits im Rahmen der Thematisierung der konstituierenden Prinzipien angeführt. 
renpotenziale, die allerdings mittels regulierender Prinzipien sozialverträglich ausgestaltet werden können. Unter die regulierenden Prinzipien ist insbesondere die Einkommenspolitik zu subsumieren, d. i. eine progressive Einkommensbesteuerung nach dem Leistungsfähigkeitsprinzip, die eine begrenzte Umverteilung nach ex ante definierten sozialpolitischen Gerechtigkeitspostulaten gestattet. Begrenzt deshalb, da zum einen eine expansive Verteilungspolitik den diskretionären Spielraum von Interessen- und Machtgruppen unkontrollierbar erhöhen würde, und zum anderen auch deshalb, da eine unverhältnismäßige Steuerpolitik die Investitionsneigung der Wirtschaftssubjekte drastisch reduzieren würde. Des Weiteren zählt zu den regulierenden Prinzipien die Korrektur negativer externer Effekte z. B. in Bezug auf die sozioökologische Nachbaltigkeit sowie die Vermeidung des working poor. Letzteres impliziert die staatliche Gewährleistung eines Mindestmaßes an sozialer Absicherung (und u. U. Mindestlöhne (vgl. Eucken 1952/2004: 304; Dietze/Eucken/Lampe 1943/2008: 110)) - bei gleichzeitiger Wahrung bzw. Betonung des Primats der (anreizkompatiblen) Eigeninitiative und der Selbstverantwortung (vgl. Eucken 1952/2004: 319). Dies wiederum bedeutet, dass der Sozialstaat gemäß dem Subsidiaritätsprinzip erst in existenziellen (und unverschuldeten) Notlagen, in solchen also, wo das Existenzminimum bzw. ein gewisser Mindestlebensstandard gefährdet ist, eingreifen soll (d. i. Abgrenzung Sozial-vs. Woblfabrtsstaat). Eucken betont unermüdlich die Komplementarität zwischen den konstituierenden und regulierenden Prinzipien auf der einen und der sozialen (Tausch- und Verteilungs)Gerechtigkeit ${ }^{16}$ und Humanität auf der anderen Seite. Ähnlich wie Hayek, Buchanan ${ }^{17}$ u. a. versteht auch Eucken Gerechtigkeit nicht als materiale Ergebnis- oder Endzustandsgerechtigkeit, sondern als prozedurale Regel- oder Verfabrensgerechtigkeit, da nur eine solche hinreichend konsensfähig sei und so den demokratischen Legitimationserfordernissen entspräche. Außerdem ist eine derartige, primär am Gemeinwohl orientierte Gerechtigkeitskonzeption nur bedingt partikularistischer Einflussnahme ausgesetzt.

Bemerkenswert ist Euckens Diskussion der Sozialpolitik auch in anderer Hinsicht: In seinem Grundsätze-Kapitel über Sozialpolitik spricht er eine ganze Reihe von hochaktuellen wirtschaftsethischen Fragestellungen an, so u. a. die Frage der betriebsinternen Mitbestimmung und Partizipation, die Rolle von Gewerkschaften, die Schaffung menschenwürdiger Arbeitsbedingungen, die Bedeutung von Arbeitnehmerschutzmaßnahmen, den Marxschen Aspekt der ökonomischen Exploitation ${ }^{18}$ und den Zusammenhang von Arbeit und personaler Identität. ${ }^{19}$ Diese Aspekte können und sollen hier

16 Euckens Ordnungspolitik verbindet geschickt Elemente der Tausch- und Verteilungsgerechtigkeit (vgl. hierzu Schumann/Nutzinger (2009)); s. auch Eucken (1952/2004: 317).

$17 \quad$ Vgl. zu den persönlichen und inhaltlichen Verbindungslinien zwischen Eucken, Hayek und Buchanan sowie zur Kompatibilität zwischen NIÖ, Constitutional Political Economy und Freiburger Lehrstubltradition: Goldschmidt/Wohlgemuth (2008); Vanberg (2000) und (2004) sowie Leipold (1990).

18 Vgl. Eucken (1952/2004: 322): „Um Ausbeutung zu verhindern, ist der Vermachtung entgegenzuwirken. Zwischen den Partnern sollte Gleichgewicht herrschen.“ (s. auch a. a. O.: 44ff.)

19 Siehe Eucken (1952/2004: 317f. und 323). Dort heißt es u. a.: „Vermeidung der Arbeitslosigkeit ist ein zentrales Problem der Wirtschaftspolitik auch im Hinblick auf die Erhaltung der Freiheit.“ 
nicht eingehender thematisiert werden;20 worauf es an dieser Stelle ankommt, ist die Tatsache, dass all diese Punkte Euckens überragendes Interesse an der Etablierung einer ethisch-normativen Ordnung, die den Grundgedanken der Humanität und Gerechtigkeit verpflichtet ist, verdeutlichen:

„Wenn ein Bestreben, das der Ordnung und der Freiheit zugleich gerecht werden will, das alltägliche Handeln (...) bestimmt, dann ist der Aufbau einer freien Ordnung möglich, und man dient dem, was der großen deutschen und europäischen geistigen Tradition entspricht, der Humanität" (Eucken 1952/2004: 324).

\section{Das Euckensche Freiheitsverständnis}

Das Euckensche Freiheitsverständnis ist vor dem Hintergrund des Spannungsverhältnisses von Freiheit, Herrschaft, Macht und Ordnung zu sehen. Die Ausübung von Freiheit, verstanden als ein Maximum an Wahl- und Selbstverwirklichungsmöglichkeiten, sowie die Wahrnehmung gewisser formeller Rechte sind durch sozioökonomische Machtstrukturen, die stets verbunden sind mit Abhängigkeitsbeziehungen und Unfreiheit, bedroht; ${ }^{21}$ sie können allerdings durch adäquate Ordnungsprinzipien und den bewussten Verzicht auf prozesspolitische Interventionen geschützt werden. So könnte man das Euckensche liberale Credo pointiert zusammenfassen. Fundamental ist diesbezüglich die aufklärerische Autonomiekonzeption Kants ${ }^{22}$ sowie Euckens persönliche Auseinandersetzung mit totalitären Ideologien. ${ }^{23}$

Die innere und äußere Freiheit des Menschen, ${ }^{24} \mathrm{~d}$. h. seine Autonomie und seine Fähigkeit zum mündigen moralischen Handeln, sind gemäß (Kant ${ }^{25}$ und) Eucken in

20 An dieser Stelle sei darauf verwiesen, dass das Grundsätze-Kapitel zum Thema Sozialpolitik aufgrund des frühen Todes Euckens unvollendet bleiben musste. Dies ist umso bedauerlicher, da gerade jener Abschnitt wirtschaftsethisch bedeutsames Material enthält.

21 Siehe Eucken (1952/2004: 174): „Besitz von Macht provoziert Willkürakte, gefährdet die Freiheit anderer Menschen, zerstört gewachsene und gute Ordnungen.“

Vgl. hierzu Kant (1977a) und (1977b). Euckens Rekurs auf Kant und andere Philosophen (vgl. Nawroth 1961/1962: 76f.) entkräftet Ulrichs zu Beginn geäußerte Kritik; gleichzeitig rückt sie Euckens Konzeption selbst in die Nähe der Integrativen Wirtschaftsethik.

Vgl. Eucken (1952/2004: 318): „Eine gut funktionierende Wettbewerbsordnung vermag den Menschen nicht bloß vor wirtschaftlicher Not zu bewahren. Sie allein schützt ihn vor der Gefahr des Totalitarismus.“

$24 \quad$ Freiheit ist einer der zentralen Termini in der (Kantischen) Argumentationskette Euckens; sie ist konstitutiv für die Menschwerdung (vgl. Eucken (1948: 73) und (1952/2004: 176 und 369)): „Ohne Freiheit, ohne spontane Selbsttätigkeit ist der Mensch nicht ,Mensch“،

Eucken beruft sich häufig auf Kant, wenn es um die philosophische Autorisierung seines Freiheitskonzeptes geht (vgl. Eucken (1952/2004: 176 und 360)): „Nach (Kants) Auffassung ist es die Aufgabe des Staates, eine Form zu finden, in der ein (...) Zusammenleben und zugleich ein größtmöglicher Spielraum für die freie Entfaltung der individuellen Kräfte möglich ist. Die absolute Freiheit des Naturzustandes soll durch Gesetze eingeschränkt werden, durch die der einzelne gegen die Willkür anderer geschützt wird. Aber andererseits soll doch die freie Betätigung der vielen einzelnen im Wettbewerb miteinander die Gesellschaft fördern." Die diskursiven Parallelen zwischen Kant und Eucken sind offensichtlich; vgl. zu Kant und Eucken: Schumann/Nutzinger (2009) und Oswalt (2001). 
dreifacher Weise bedroht (vgl. Eucken 1952/2004: 177): durch die Macht der Privatunternehmen, durch die halböffentliche Macht der gesellschaftlichen Kollektive und durch die Macht des Staates, der sich mit privaten Machthabern verbinden kann, womit die Risiken eines Machtmissbrauchs und der Gruppenanarchie (vgl. Eucken 1952/2004: 79; 2001: 16) potenziert werden. Um den genannten Bedrohungsszenarien zu entgehen und um eine umfassende individualethische Freiheit realisieren zu können, ist der liberale Rechtsstaat (in Kombination mit den bereits angeführten Grundsätzen der Wirtschaftspolitik und den konstituierenden und regulierenden Prinzipien) von essenzieller Bedeutung:

„Der Staat soll unter dem Recht stehen. (...) Deshalb soll er die Freiheits- und Rechtssphären der (...) Bürger anerkennen und schützen. (...) Dabei hat der Rechtsstaat das Recht seiner Bürger gegen zwei Seiten hin zu sichern: gegen die (willkürliche) Zwangsgewalt staatlicher Verwaltungsorgane, die in aller Geschichte (...) die Tendenz haben, in angeblich öffentlichem Interesse die persönliche Freiheit zu verletzen; zugleich aber gegen die Bedrohung der Bürger gegeneinander (d. i. Übergriffe anderer Privater in die individuelle Freiheitssphäre).“26

Eucken tritt für Gewaltenteilung und verfassungsmäßig garantierte Grundrechte ein; explizit werden von ihm die Meinungsfreiheit, die geistige Freiheit des Denkens und der Kritik, das Recht auf freie Wohnungs- und Arbeitsplatzwahl, das (relative) Recht auf Vertrags- und Gewerbefreiheit, die Unantastbarkeit des Privateigentums und das Recht auf Freiheit der Person angeführt (vgl. Eucken 1952/2004: 48, 125, 130ff). Bemerkenswert ist Euckens Verknüpfung von Freiheit und politökonomischer Ordnung, von Freiheit und Freiheitsbeschränkung durch Exploitation und ökonomistische, d. h. monopolartige Machtstrukturen. ${ }^{27}$ Derart mutiert Freiheit in totalitäre Überwachung, Willkür und Zwang, werden sozioökonomische Abhängigkeitsverhältnisse etabliert und legitime Freiheitsrechte durch einen Missbrauch schrankenloser Freiheit gefährdet. ${ }^{28}$

Hinzu kommt ein Aspekt, der auf Großmann-Doerth, einem Gründungsmitglied der Freiburger Schule, zurückzuführen ist: das selbstgeschaffene Recht der Wirtschaff, ${ }^{29}$ das nicht nur neben staatlich gesetztes Recht tritt, sondern jenes in weiten Teilen gar bereits

Eucken (1952/2004: 48); vgl. (a. a. O.: 128ff. und 175ff.) sowie Eucken (2001: 21). Die Freiheitssphäre muss durch das Recht gesichert werden; Freiheit und Recht sind eng miteinander verzahnt: „Diese Freiheitssphäre hat das Recht zu sichern. (...) Sowenig der Mensch, der in dieser Ordnung steht, seine eigene Freiheit selbst aufheben darf, sowenig darf er die Freiheitssphäre der anderen mißachten. Hier an der Freiheitssphäre der anderen findet seine Person ihre Grenzen. Indem er diese Freiheitssphäre achtet, übt er Humanität. Freiheit - richtig verstanden - und $\mathrm{Hu}-$ manität und Recht gehören zusammen, sind untrennbar miteinander verbunden. “ (Eucken 1952/2004: 176; vgl. Kant (1977a: 337f.))

„Wo Monopole (...) bestehen, ist die persönliche Freiheit (und die Konsumentensouveränität) (...) sehr beschränkt.“ (Eucken (1952/2004: 49); vgl. auch (a. a. O.: 163))

28 „(..) daß die Gewährung von Freiheit eine Gefahr für die Freiheit werden kann, wenn sie die Bildung privater Macht ermöglicht (...).“ (Eucken 1952/2004: 53)

29 Vgl. Großmann-Doerth (1933/2008), Eucken (1950/1965: 56) sowie Eucken (1952/2004: 51f., 295 und 328f.). 
verdrängt hat. Eucken zufolge ist die grundsätzliche Gefahr der sog. Selbstverwaltungskörperschaft bzw. der Berufsstände darin zu sehen, dass diese nur partikulare Interessen vertreten, dass sie zur Monopolisierung und Kartellbildung neigen und dass sie die Gefahr der Tyrannis und Gruppenanarchie erhöhen (und so die Freiheit der Individuen erheblich einschränken). Darüber hinaus führt der zunehmende Einfluss von privilegierten und mit Sonderrechten ausgestatteten Lobbyisten in Kombination mit dem selbstgeschaffenen Recht der Wirtschaft zu einer „Zunahme im Umfange der Staatstätigkeit (d. i. zunehmende Zentralisierung und Bürokratisierung) und die gleichzeitige Abnabme der staatlichen Autorität" (Eucken 1952/2004: 327): Der Staat gerät in wachsende Abhängigkeit von Macht- und Interessengruppen, die außerhalb der Verfassung stehen und über nur bedingte demokratische Legitimation verfügen und die bestrebt sind, nur ihre kurzfristigen Einzelinteressen durchzusetzen. Der Handlungsspielraum des Staates wird stetig eingeschränkt, die verfassungsmäßigen Gewalten werden entthront und die Willensbildung der Politik massiv von Gruppeninteressen beeinflusst. Letztlich kann es zu einem Zerfall, zu einer Umformung des Staates und zu einer Aushöhlung seiner politbürokratischen Kompetenzen kommen - mit nachteiligen Auswirkungen auf die Freiheitsrechte der Bürger. ${ }^{30}$ Liberal-rechtsstaatliche Prinzipien und Menschenrechte lassen sich also nur dort verwirklichen, wo es gelingt, diese institutionell so zu verankern, dass Macht- und Herrschaftsstrukturen, seien diese nun politischer, sozialer oder ökonomischer Natur, möglichst minimiert werden ${ }^{31}$ - und dies ist eben primär nur in einer sozial verpflichteten Wettbewerbsordnung möglich, die auf den von Eucken entwickelten konstituierenden und regulierenden Grundsätzen fußt (vgl. diesbezüglich Eucken 1952/2004: 52).

Zentralverwaltungswirtschaften werden neben ihren ökonomischen (d. i. Gleichgewichtslosigkeit, Disproportionalitäten, Wissensanmaßung, Anreizproblematik, fehlende Adaptionsfähigkeit, Versorgungsminima u. ä.) insbesondere wegen ihrer sozialliberalen Defizite abgelehnt: ${ }^{32}$ Koordinationsmechanismen (d. i. Preise als Informationsund Knappheitsanzeiger wie in einer Verkehrswirtschaft üblich) werden hier durch dirigistische Subordinationsmechanismen ersetzt. Auch mit Blick auf die Überwindung der Sozialen Frage und die Herstellung sozialer Gerechtigkeit bleibt die Planwirtschaft defizitär. ${ }^{33}$ Aber auch libertäre und Laissez-faire-Wirtschaftssysteme sind in freiheitlicher Hinsicht unzureichend, da sie der systemimmanenten Tendenz zur Vermachtung

Ist der Staat erst einmal zum Spielball der Interessengruppen geworden, so zieht dies unweigerlich eine gesellschaftliche „Vermassung“ und eine Entpersönlichung bzw. „Auslöschung der Persönlichkeit" nach sich (Eucken (1932a); vgl. auch Eucken (1932b) sowie Eucken (1952/2004: 337)).

31 Eucken geht es nicht nur um die Minimierung von politökonomischen Machtstrukturen. Eigentliches Ziel seines ersten wirtschaftspolitischen Grundsatzes ist die Prävention der Evolution von Macht ausübenden Systemen überhaupt (vgl. Eucken (1952/2004: 172ff.) sowie Eucken (2001: 17 und 83)).

Vgl. zur Ballung ökonomischer Macht und zur Einschränkung individueller Freiheit und Autonomie innerhalb einer Zentralverwaltungswirtschaft: Eucken (1950/1965: 86f. sowie 196ff.).

33 Eine vergleichbare Argumentation entwickelt auch Hayek (1971), der neben den ökonomischen Anreizproblemen gleichfalls auf die keineswegs befriedigende Lösung der Sozialen Frage bzw. von Verteilungsfragen im Rahmen planwirtschaftlicher Systeme hinweist. 
und Monopolbildung nicht adäquat begegnen. ${ }^{34}$ Einzig eine wettbewerblich organisierte und sozial verpflichtete Wirtschaftsordnungspolitik ist, so Eucken, in der Lage, ökonomische Funktionsfähigkeit mit der Anerkennung der Menschenwürde und der Förderung von Gerechtigkeit und Humanität zu paaren. Eucken bemerkt zur Interdependenz seiner Zielgrößen:

„Damit aber stößt man auf den Kernpunkt der sozialen Frage: die Freiheit. (...) Die Herrschaft (...) (der) Machtkörper gefährdet die Durchsetzung der Gerechtigkeit, und mangelnde Sicherheit entspringt aus mangelnder Freiheit. Ohne Freiheit der Person die soziale Frage zu lösen, ist unmöglich." 35

Menschen sind für Eucken immer auch Selbstzweck; 36 eine Instrumentalisierung der Menschen ausschließlich als Mittel zum Zweck kommt für Eucken nicht in Frage ein direkter Bezug auf die Selbstzweckformel des Kategorischen Imperativs. Euckens Eintreten für Wettbewerb und möglichst geringe Markteintrittsbarrieren resultiert letztlich aus der Einsicht, dass nur in einer Ordnung der vollständigen Konkurrenz, innerhalb derer nur bedingt Marktmachtpositionen existieren, Freiheits- und Menschenrechte garantiert werden können. Eucken ist also bestrebt, Ordnungsformen zu implementieren, die maximale Freiheit ${ }^{37}$ gewähren, die Freiheitsmissbräuche einschränken, die das Selbstbestimmungsrecht der Bürger verwirklichen helfen und die somit einen Beitrag zur Überwindung der Unmündigkeit leisten (vgl. Eucken 1948: 73ff.; Böhm 1950: XXXV).

Schließlich sei noch auf Euckens persönliches Engagement für Freiheit, Menschenrechte und Demokratie und gegen totalitäre und autokratische Regimes eingegangen..$^{38}$ Eine Einordnung der Hauptwerke Walter Euckens in den historischen Kontext ist

Eucken (2001: 14) schreibt: „Das Recht der Vertragsfreiheit durfte (in der Zeit des laissez faire) (...) dazu benutzt werden, um Konkurrenz zu beseitigen und (...) die Freiheit anderer zu beschränken."

Eucken (1952/2004: 126); s. Eucken (2001: 45) sowie zur Interdependenz von Freiheit und Überwindung der Sozialen Frage: Eucken (1952/2004: 189, 193 und 370).

Dies zeigt nicht zuletzt seine an Kant (Rudolf Eucken und den Neukantianismus respektive Neoidealismus) angelehnte (wirtschafts-)ethische Argumentation sowie sein alltäglich-konkretes Eintreten für Humanität und Liberalität (vgl. Nawroth (1961/1962: 15) sowie (1965: 11 und 94ff.)). Vgl. weiterhin Eucken (1948: 76) („,...) daß es hier um den Menschen als Menschen geht") sowie seine Kritik an dem alles durchdringenden, totalitären Wirtschaftsstaat (vgl. a. a. O.: 75ff.): „Der einzelne Mensch wird (hier) zur Sache und verliert den Charakter als Person. Der Apparat ist Zweck, der Mensch Mittel.“ (a. a. O.: 75) Abschließend fragt Eucken: „Ist eine Wirtschaftsordnung möglich, in der die Menschen nicht nur Mittel zum Zweck, nicht also nur Teilchen des Apparates sind?" (a. a. O.: 77)

37 Die Freiheit des Einzelnen findet, gemäß der Kantischen Freiheitsdefinition, dort ihre Grenzen, wo die Freiheit des Anderen beginnt (vgl. Kant (1977a: 337f.)). „Wie der Rechtsstaat so soll auch die Wettbewerbsordnung einen Rahmen schaffen, in dem die freie Betätigung des einzelnen durch die Freiheitssphäre des anderen begrenzt wird und so die menschlichen Freiheitsbereiche ins Gleichgewicht gelangen. - In der Tat ist der Wille zur Wettbewerbsordnung mit dem Willen zur Freiheit verbunden.“ (Eucken 1949: 27)

38 Für weiterführende Informationen vgl. Blumenberg-Lampe (1973), Rieter/Schmolz (1993), Goldschmidt (1997) und (2005) sowie als Gegenposition Haselbach (1991) und Ptak (2004: 62ff.). 
unerlässlich für ein adäquates Verständnis seiner wirtschaftspolitischen Empfehlungen: Eucken zählte neben Lampe, von Dietze und Ritter zum Kern der Freiburger Widerstandsbewegung gegen das NS-Regime. Die sog. Freiburger Kreise setzten sich zusammen aus dem Freiburger Konæil (gegründet 1938 nach den Novemberpogromen) dem Freiburger Bonhoeffer Kreis und der Arbeitsgemeinschaft Erwin von Beckerath. Eucken besuchte zudem regelmäßig das Diehl-Seminar Der Einzelne und die Gemeinschaft, er war Mitglied der Bekennenden Kirche ${ }^{39}$ und einer der schärfsten universitätsinternen Opponenten des Rektorats von Heidegger mitsamt seiner Affinität zur NSGleichschaltungspolitik und zum Führerprinzip. Eucken selbst vertrat in leidenschaftlicher Weise seine ordoliberale Position auch und gerade während des Dritten Reiches - erinnert sei an dieser Stelle an die Volkswirtschaftsfibel (vgl. Dietze/Eucken/Lampe 1941/1942), die er zusammen mit von Dietze und Lampe verfasste und die sich mit einer christlich-ordoliberalen Nachkriegswirtschaftsordnung beschäftigt; weiterhin sei erinnert an Euckens den Idealen der Aufklärung verpflichtete Vorlesungsreihe Kampf der Wissenschaft (1936), in der er sich mit der Philosophie Sokrates', Spinozas und Galileis auseinander setzte. Auch sein Eintreten für in Folge der NS-Rassegesetze diskriminierte Bevölkerungsgruppen soll hier nicht unerwähnt bleiben (und das obwohl Euckens Schwiegermutter Jüdin, seine Frau Edith Halbjüdin und er gemäß den NSRassegesetzen als „nichtarisch-versippt“ eingestuft wurde): So setzte sich Eucken für das alltägliche Wohlergehen der befreundeten Familie Husserl ein. Dies alles provozierte naturgemäß Reaktionen der totalitären nationalsozialistischen Machthaber: Mehrfach erhielt Eucken Morddrohungen (ab 1936), die zweite Auflage seines Werkes Nationalökonomie wozu? wurde verboten (1938) und nach dem missglückten Attentat vom 20. Juli 1944 wurde Eucken unter Folterandrohung verhört und kurzzeitig interniert (vgl. Oswalt 2005: 332ff.; 2008: 119ff.).

\section{Die Grenzen der Staatstätigkeit}

Eucken geht es nicht um eine quantitative Bestimmung der Staatsaufgaben; vielmehr ist er bestrebt, das qualitative Aufgabenspektrum und die Grenzen der Staatstätigkeit und Staatsmacht klar zu definieren:

„Der Staat hat die Formen, in denen gewirtschaftet wird, zu beeinflussen, aber er hat nicht den Wirtschaftsprozeß selbst zu führen. (...) Staatliche Planung der Formen - ja; staatliche Planung und Lenkung des Wirtschaftsprozesses - nein" (Eucken 2001: 77). Der zweite Grundsatz der Wirtschaftspolitik lautet folglich: „Die wirtschaftspolitische

39 Die normativ-metaphysische Einbettung der Werte Freiheit und Humanität lässt sich auch an Euckens (christlicher) Religiosität festmachen. Diese gipfelt in der Selbstaussage: „Ich aber könnte weder existieren noch arbeiten, wenn ich nicht wüsste, dass Gott existiert." (zitiert nach Lenel (1991)) In dem Aufsatz Religion - Wirtschaft - Staat (Eucken (1932a)) konstatiert Eucken eine tief greifende gesellschaftliche Sinnkrise und ein fundamentales ethisches Vakuum. Nihilistischanarchische Sinnkrise und Wertevakuum können gemäß Eucken nur mit Hilfe von (christlicher) Religion überwunden werden; nur die Religion als (mögliche) ordnende Potenz ist fähig, einen metaphysischen Reformations- und Erneuerungsprozess in Gang zu setzen. 
Tätigkeit des Staates sollte auf die Gestaltung der Ordnungsformen der Wirtschaft gerichtet sein, nicht auf die Lenkung des Wirtschaftsprozesses. "40

Ziel der wettbewerblich organisierten Wirtschaftsordnung ist ein harmonischer Ausgleich von „ökonomischer Sachgesetzlichkeit“ und „,soziale(m) und ethische(m) Ordnungswollen“" (Eucken 1952/2004: 370), von Einzel- und Gesamtinteresse. ${ }^{41}$ Eucken ist sich sehr wohl bewusst, dass Machtstrukturen jeglicher Art einen Ausgleich von Einzelinteresse und Gemeinwohl ebenso verhindern wie eine Laissez-faire-Ordnung (vgl. Eucken 1952/2004: 359f.). Daher plädiert Eucken tendenziellt2 für eine gesetztte, konstruktivistische Ordnung im Gegensatz zur von Hayek favorisierten gewachsenen, evolutionären Ordnung (vgl. Eucken 1952/2004: 372ff; Hayek 1970/1996) und ihrem Vertrauen auf evolutionäre Spontaneität und die Selbstverwirklichung des ordre naturel. Spezifisch ordoliberal sind die Unterscheidung von Ordnungs- und Prozesspolitik, die Trennung von Spielregeln und Spielzügen und die Selbstbeschränkung des Staates auf die Setzung des Ordnungsrahmens. ${ }^{43}$ Die Unfähigkeit des Staates, den Wirtschaftsprozess selbst zu führen, resultiert zum einen aus den vielfältigen Informations-, Wissens- und Anreizrestriktionen (d. i. Informationsasymmetrien und Interessenkollisionen); andererseits unterliegt die staatliche Prozesspolitik - insbesondere in Form eines punktuellen Interventionismus (vgl. Eucken 1952/2004: 195f., 221, 306; 1932b: 303f.) - der Einflussnahme von Macht- und Gruppeninteressen. Damit sind die Staatsaufgaben klar umrissen: Der Staat als Ordnungsinstanz soll sich auf die Gestaltung der Formen konzentrieren, jedoch keine eigenständige und unmittelbare Lenkungspolitik durch laufende Eingriffe betreiben.

Ein wichtiger, bei Buchanan (1984) wiederzufindender Grundsatz der Rechtsstaatstätigkeit ist das Neutralitätsgebot bzw. Diskriminierungsverbot. Der Staat hat auf strikte Privilegienfreiheit und Unabhängigkeit von Partikularinteressen zu achten. Er ist dem Gemeinwohl verpflichtet und hat die Rechte seiner Bürger zu schützen. Vor allem hat er für gleiche und faire Start-, Teilhabe- und Selbstverwirklichungschancen der Individuen (d. i. formale Rechts- und Chancengleichheit), für maximale Freiheit und für die Wahrung der Menschenwürde Sorge zu tragen. Euckens antitotalitäres und antiautoritäres „Programm der Freiheit“ (Eucken 1952/2004: 370) lässt sich also wie folgt zusam-

$40 \quad$ Eucken (1952/2004: 336); s. zur Unterscheidung von Form und Prozess auch: (a. a. O.: 242). Der Staat besitzt jedoch keine absolute Gestaltungsfreiheit: „,...) es besteht nicht die Freiheit, die Spielregeln oder die Formen, in denen sich der Wirtschaftsprozeß abwickelt (...), nach Willkür zu gestalten."

41 Vgl. zur Kanalisierung des Eigeninteresses via Institutionenordnung: Eucken (1952/2004: 365); vgl. (a. a. O.: 368).

42 Gemäß Eucken ist es die Aufgabe des Rechtsstaates, die Freiheitssphäre der Individuen zu sichern. Der (konstituierenden und regulierenden) Wirtschaftsordnungspolitik kommt die Aufgabe zu, die natürliche, gottgewollte Ordnung zu verwirklichen (vgl. Eucken (1948: 73)). Ohne staatliche Mithilfe ist die Realisierung des natürlichen Ordos allerdings wenig chancenreich. Insofern ist von einer „konstruktivistischen“ respektive ,gesetzten“ Gesellschaftsordnung auszugehen.

43 Vgl. Eucken (1952/2004: 250): ,Wie der Rechtsstaat, so soll auch die Wettbewerbsordnung einen Rahmen schaffen, in dem die freie Betätigung des einzelnen durch die Freiheitssphäre der anderen begrenzt wird und so die menschlichen Freiheitsbereiche ins Gleichgewicht gelangen. - In der Tat ist der Wille zur Wettbewerbsordnung mit dem Willen zur Freiheit eng verbunden.“ 
menfassen: Verbinderung von Macht und Ermöglichung von Freibeit:44 Ebenfalls in Anlehnung an Buchanan (1984) könnte man bei Euckens Staatskonzeption eine rechts- und eine leistungsstaatliche Komponente differenzieren. Letztere wird von den vier regulierenden Prinzipien ausgefüllt - Prinzipien, die für eine Korrektur bei Marktversagen sorgen sollen. Aber nicht nur Marktversagen wird im Ordoliberalismus korrigiert; auch Staatsversagen wird bei Fokussierung auf die Ordnungspolitik minimiert, da prozesspolitisches Staatsversagen per definitionem limitiert ist. Bemerkenswert ist schließlich Euckens Eintreten für eine transparente, nachbaltige und staatlich regulierte Wirtschaftsordnung, in der der Wettbewerb als Verfahren gesellschaftlicher Machtkontrolle respektive als „Entmachtungsinstrument ${ }^{\star 45}$ fungiert.

\section{Eucken im Spannungsfeld von Moralökonomik und Integrativer Wirtschafts- ethik ${ }^{46}$}

Gemäß der moralökonomischen Argumentation von Homann ist der systematische Ort der Moral die Rahmenordnung (vgl. Homann/Blome-Drees 1992: 35); moralische Imperative sind idealiter in den Spielregeln inkorporiert. Die Spielzüge, d. h. die Marktabläufe und transaktionen sind grundsätzlich moralfrei. Ausgehend von dem Homo-oeconomicusModell werden Marktprozesse als Dilemmata und unter institutionellen Gesichtspunkten analysiert. Primäres Ziel ist die Etablierung von erwünschten und die Überwindung unerwünschter Dilemmasituationen mittels einer anreizkompatiblen Rahmenordnung. Die externen institutionellen Handlungsbedingungen determinieren bzw. kanalisieren die individuellen Handlungen (vgl. Homann/Lütge 2004/2005; Pies 2002; Suchanek 2001/2007). Auch Eucken unterstützt die Separierung zwischen konstitutioneller (Wirtschaftsverfassung) und postkonstitutioneller Ebene (Wirtschaftsprozess), von Spielregeln und Spielzügen, die die "Ökonomische Ethik" (Suchanek 2001/2007) vornimmt; seine Argumentation kann mit Dilemmastrukturen illustriert werden. Auch sieht er die Notwendigkeit, Wettbewerbsmechanismen zum Vorteil aller via Anreize kanalisierender (vgl. Eucken 1952/2004: 360) Institutionenordnung zu etablieren und für konsensfähige konstitutionelle Regelinteressen (d. i. Kriterien der Unparteilichkeit, Universalisierbarkeit und freiwilligen (Regel-)Selbstbindung) und gegen Privilegieninteres-

44 D. i. eine Marktwirtschaft frei von staatlicher Willkür und frei von privater Wirtschaftsmacht.

45 Vgl. Böhm (1961: 22); s. auch Eucken (2001: 83): „Kontrolle durch Wettbewerb“. Im Vorwort zur ersten Ausgabe des Ordo-Jahrbuchs wird zudem deutlich, dass die Autoren Wettbewerb als Mittel, nicht aber als letzten Zweck definieren (vgl. Böhm/Eucken (1948: XI)).

46 Im nachfolgenden Rekurs geht es primär um einen direkten Vergleich, nicht aber um eine umfassende Darlegung der Konzeptionen Euckens, Homanns und Ulrichs (dabei beschränken wir uns der Einfachheit halber auf die Werke von Ulrich und Homann und verzichten auf eine eingehende Auseinandersetzung mit den z. T. deutlich voneinander abweichenden Positionen der Homann-Schüler). Neben dem Aufzeigen möglicher Parallelen und Unterschiede der verschiedenen Theorien und einer Relativierung der Kritikpunkte Ulrichs am Ordoliberalismus Euckenscher Prägung ist es das Bestreben des nachfolgenden Abschnitts das (vermittelnde) wirtschaftsethische Potenzial der Schriften Euckens hervortreten zu lassen. 
sen einzutreten. Schließlich besteht auch eine nicht bloß terminologische ${ }^{47}$ Einigkeit dahingehend, dass eine adäquate institutionelle Rahmenordnung bzw. Wirtschaftsordnungspolitik Freiheit überhaupt erst ermöglicht. Eucken ist also durchaus ein Vorläufer der NIÖ.

Jedoch lassen sich auch eine Reihe von Abweichungen in der inhaltlichen Argumentation kenntlich machen: So betont Homann im Gegensatz zur Kleingruppengesellschaft mit ihren Face-to-face-Beziehungsstrukturen die Makro- und Mesoebene einer anonymen und globalisierten Großgruppengesellschaft mit ihren formalisierten Sanktionsmechanismen, während die Mikro- oder Individualebene und kommunitaristische und gemeinschaftsbezogene Aspekte weitestgehend negiert werden. Eucken hingegen versucht den Spagat zwischen individualethischer Mikro-, unternehmensethischer Mesound formell-institutionenethischer Makroebene, indem er ganz bewusst Kantisches Gedankengut in seinen Ansatz integriert. Zentrale Euckensche Termini sind Ordnungspolitik und Wettbewerbsordnung, aber eben auch Freiheit, Autonomie und Selbstverantwortung. Eucken sympathisiert nicht nur mit der Kantischen „Gesinnungsethik" - ganz im Gegensatz zur Moralökonomik - ; er würde sehr wahrscheinlich gleichfalls eine funktionalistische Begründung respektive einen instrumentellen Gebrauch der Moral, wie ihn z. B. Homann zu favorisieren scheint, rundweg ablehnen. Die Moral ist eben kein Schmiermittel der Ökonomie; auch kommt es nicht ausschließlich auf die Funktionsfähigkeit einer Wirtschaftsordnung (d. i. Primat der Ökonomik) an, sondern gleichsam auch auf ihre Humanität, (Verfahrens- und Verteilungs-) Gerechtigkeit und sozioökologische Nachhaltigkeit. Ein deontologisches bzw. philosophisch-normatives Defizit, wie es von Ulrich den moralökonomisch argumentierenden Autoren vorgeworfen wird (vgl. Ulrich 1997/2008: 112ff., 389) ist in den Werken Euckens unseres Erachtens daher nicht zu finden (vgl. Wörsdörfer 2010).

Ulrich hingegen hat eine andersartige Herangehensweise an wirtschaftsethische Problemstellungen. Er knüpft mit seinem St. Galler Ansatz, der Integrativen Wirtschaftsethik (vgl. Ulrich 1994; 1997/2008; 2004) - ähnlich wie Eucken - unmittelbar an Kant (und die Diskursethik) an und unterstreicht die Bedeutsamkeit des Humanitätselements, um die Begrifflichkeit Euckens erneut aufzugreifen, während Homann seinen Fokus verstärkt auf Funktionalitätsgesichtspunkte richtet. Aber auch hier findet sich eine Einseitigkeit hinsichtlich der Relation von Gemeinwohl und Eigeninteresse sowie von Ethik und Ökonomik, die sich derart bei Eucken nicht konstatieren lässt, denn auch Ulrich bleibt - mit umgedrehten Vorzeichen - defizitär, was den Kriteriendualismus anbelangt: Das Primat der Ökonomik (Homann) wird ersetzt durch ein Primat der Ethik (vgl. Ulrich 1997/2008: 129ff.); eine wirkliche Integration von Ethik und Ökonomik wie von Eucken postuliert misslingt jedoch.

Ulrich kritisiert den ökonomischen Imperialismus, das „ökonomische( Sachzwangdenken“ und die „Metaphysik des Marktes“ mit ihrer quasi-religiösen und ideologischen Aufladung der reinen ökonomischen Vernunft. Er fordert eine Transformation der ökonomischen Vernunft (d. i. „Lebensdienlichkeit“ der Ökonomie und „Vernunftethik kabular der Freiburger Schule und des Ordoliberalismus i. w. S. zurück (vgl. die angegebene Literatur). 
des Wirtschaftens“ bzw. ethisch-kommunikative Rationalität), eine Überwindung der Zwei-Welten-Konzeption von Ethik und Ökonomik und eine Reintegration der ethischen Lebens- und der ökonomischen Systemwelt. Als deontologische oder „wirtschaftsbürgerliche Minimalethik“ (Ulrich 1997/2008: 142, 177, 11, 14, 347) definiert er einen republikanischen Liberalismus, in dem das Individuum nicht Bourgeois („Besitzbürger"), sondern Citoyen sei und in dem ein zivilgesellschaftliches „Wirtschaftsbürgerethos" herrsche (d. i. umfassender Bürgerstatus und Wirtschaftsbürgerrechte ${ }^{48}$ ). Eucken hätte einer derartigen (Wirtschafts-)Bürgergesellschaft bzw. einem Kantischen Republikanismus durchaus etwas abgewinnen können:49 Das der republikanisch-liberalen Konzeption zugrunde liegende Menschenbild - der Mensch als freier, vernünftiger und mündiger Staatsbürger, ausgestattet mit einer Vielzahl an politökonomischen Partizipationsrechten - ist mit demjenigen Euckens, aufgrund ihres gemeinsamen philosophischen, sprich Kantischen Ursprungs, artverwandt. Insofern ist die von Ulrich angeführte Kritik, die Theorie Euckens sei ein korrektiver wirtschaftsethischer Ansatz und sie entbehre jeglicher philosophisch-normativer Fundierung, fragwürdig. ${ }^{50}$

Hinzu kommt, dass Eucken mit den philosophischen Diskursen seiner Zeit sehr vertraut war. ${ }^{51}$ Aufgewachsen in einer Künstler- und Gelehrtenfamilie beschäftigte er sich zeitlebens mit ethischen Fragestellungen. Privat ausgebildet und nachhaltig geprägt durch seinen Vater, den Philosophieprofessor und Literaturnobelpreisträger Rudolf Eucken, später dann (in Zusammenarbeit mit seiner Frau Edith, ebenfalls Philosophin und Ökonomin) Herausgeber und Essayist der weltanschaulich-philosophischen Zeitschrift Der Euckenbund bzw. Die Tatwelt und schließlich eine enge (philosophische) Freundschaft mit dem Phänomenologen Husserl - dies und eine ganze Reihe weiterer biografischer Hinweise zeigen Euckens über die Ökonomie hinausgehendes Interesse. Insbesondere interdisziplinäre Probleme vor dem Hintergrund der Zäsuren im Leben der Menschen in der Moderne, der Industrialisierung ${ }^{52}$ und der Französischen Revolution standen im Zentrum seiner Arbeits- und Forschungsschwerpunkte.

$\mathrm{Zu}$ den weiteren Parallelen zwischen Eucken und Ulrich zählen die Hinwendung zur sozioökonomischen Machtproblematik, das Postulat einer Zivilisierung und Bändigung staatlicher und ökonomischer Macht sowie das Bekenntnis zu einer sozial verpflichteten, marktwirtschaftlichen Wirtschaftsordnung. Ulrich scheint jedoch - und das unterscheidet ihn wiederum grundlegend von Eucken - das Implementationsproblem, d. i. die Frage der Umsetzung und Institutionalisierung seiner idealistischen Konzeption, zu vernachlässigen.

48

Sämtliche: Ulrich (2004: 25ff.); vgl. zur Wirtschaftsbürgerethik: Ulrich (1997/2008: 313ff.).

So schreibt Eucken (1952/2004: 199/324) beispielsweise: „Die Gesamtordnung sollte so sein, daß sie den Menschen das Leben nach ethischen Prinzipien ermöglicht.“

Auch der dritte Kritikpunkt Ulrichs (1997/2008: 378ff.), der Ordoliberalismus leiste einem Rückfall in neoklassische Denkmuster Vorschub, ist mit Blick auf Euckens Gerechtigkeitskonzeption und die von ihm vorgenommene Integration von Wirtschafts- und Sozialpolitik nicht zutreffend.

Vgl. zu Rudolf Euckens und Edmund Husserls Einfluss auf Walter Eucken: Klump/Wörsdörfer (2010).

Die mit der Industrialisierung verbundenen Problemstellungen der Sozialen Frage und des Pauperismus lassen sich Eucken zufolge nur via ordoliberale Wirtschaftsordnungspolitik beheben. 


\section{Abschließende Bemerkungen und Ausblick}

Die Werke Euckens sind für die moderne Wirtschaftsethik in vielfältiger Weise bedeutsam. Erinnert sei hier nur an folgende Bausteine seines Programms der Freiheit. der Staat als ordnende Potenz und Hüter der Wettbewerbsordnung; Fokussierung auf Ordnungspolitik und Negation der Prozesspolitik; enge Relation von leistungsfähigem Rechtsstaat, konstitutioneller Demokratie und freiheitlicher und leistungswettbewerblich institutionalisierter Wirtschaftsordnung (d. i. regulierte Selbstregulierung); Freiheit (d. h. Autonomie, Mündigkeit, kritischer Rationalismus und (Konsumenten-)Souveränität in der Tradition von Kant ${ }^{53}$ (und Smith)), Humanität und soziale Gerechtigkeit als fundamentale Kriterien; Einheit von Wirtschafts- und Sozialpolitik sowie von Freiheit und Ordnung (d. i. „Ordnen heißt in Freiheit ordnen“ (Eucken 1952/2004: 179) und Interdependenz der Ordnungen); Verhinderung von Macht und Ermöglichung von Freiheit sowie (Rudolf Euckenscher) „ethischer Aktivismus“ und „ethisches Wollen“ gepaart mit nachhaltiger ökonomischer Leistungsfähigkeit.

Diese facettenreichen Bausteine des Ordoliberalismus Euckenscher Prägung gilt es für die moderne Wirtschaftsethik - gerade vor dem Hintergrund der Finanzmarkt- und Wirtschaftskrise und des Neuen Methodenstreits - fruchtbar zu machen. Denkbar wäre beispielsweise eine Weiterführung des ordoliberalen Forschungsprogramms im Hinblick auf eine Neuausrichtung der nationalen und internationalen (d. i. europäischen) Finanz- und Wirtschaftsordnung bei gleichzeitiger Berücksichtigung und Integration (ausgewählter Aspekte) der Konzeptionen von Ulrich, Homann und der in ihrer Tradition stehenden Schüler.

Wie die Diskussion der beiden wirtschaftsethischen Ansätze von Homann und Ulrich gezeigt hat, lassen sich bei beiden sowohl Überschneidungen mit als auch fundamentale Abweichungen von der Position Euckens konstatieren. Euckens eigenständiger, interdisziplinärer Ansatz mit seiner wirtschaftsethisch bedeutsamen Verknüpfung von Philosophie und Ökonomie, von Markt und Moral scheint als Mediationsfundament für einen Dialog, für eine (Teil-)Zusammenführung von Integrativer Wirtschaftsethik und Moralökonomik prädestiniert zu sein. Dies umso mehr, als es Eucken gelingt, eine Balance von Leistungsfähigkeit und Menschenwürdigkeit, von Freiheit, (Chancen-) Gleichheit und Humanität zu präsentieren - ohne auf ein Primat der Ökonomik oder der Ethik zurückgreifen zu müssen. Eucken liefert somit einen Ausweg aus dem Zielkonflikt von Effizienz und Gerechtigkeit und ist damit sowohl in (wirtschafts-) ethischer als auch in ökonomischer Perspektive anschlussfähig.

\section{Literaturverzeichnis}

Blümle, G. (2007/2008): Freiheit und Norm bei Walter Eucken, in: ders.: Wirtschaftsgeschichte und ökonomisches Denken, Marburg, 283-306.

Blümle, G./ Goldschmidt, N. (2000): Zur Normativität ordoliberalen Denkens, in: Külp, B./ Vanberg, V. (Hrsg.): Freiheit und wettbewerbliche Ordnung, Freiburg, 15-57. 
Blumenberg-Lampe, C. (1973): Das wirtschaftspolitische Programm der ,Freiburger Kreise‘. Entwurf einer freiheitlich-sozialen Nachkriegswirtschaft, Berlin.

Böhm, F. (1937): Ordnung der Wirtschaft als geschichtliche Aufgabe und rechtsschöpferische Leistung, Stuttgart.

Böhm, F. (1950): Die Idee des Ordo im Denken Walter Euckens, in: Ordo. Jahrbuch für die Ordnung von Wirtschaft und Gesellschaft, Bd. 3, XV-LXIV.

Böhm, F. (1961): Demokratie und ökonomische Macht, in: Institut für ausländisches und internationales Wirtschaftsrecht (Hrsg.): Kartelle und Monopole im modernen Recht, Karlsruhe, $1-24$.

Böhm, F./ Eucken, W. (1948): Vorwort. Die Aufgabe des Jahrbuchs, in: Ordo. Jahrbuch für die Ordnung von Wirtschaft und Gesellschaft, Bd. 1, VII-XI.

Buchanan, J. M. (1984): Die Grenzen der Freiheit, Tübingen.

Dietze, C./ Eucken, W./ Lampe, A. (1941/1942): Volkswirtschafts-/Nationalökonomische Fibel, in: Nachlass Popitz (BA NL 1262/87), Koblenz.

Dietze, C./ Eucken, W./ Lampe, A. (1943/2008): Wirtschafts- und Sozialordnung, in: Goldschmidt, N./ Wohlgemuth, M. (Hrsg.): Grundtexte zur Freiburger Tradition der Ordnungsökonomik, Tübingen, 99-115.

Erlei, M./ Leschke, M./ Sauerland, D. (1999/2007): Neue Institutionenökonomik, Stuttgart.

Eucken, $W$. (1926): Die geistige Krise und der Kapitalismus, in: Die Tatwelt, Jg. II, Heft 1/3 (Januar/März), 13-16.

Eucken, W. (1930): Wirtschaftsentwicklung contra Kulturentwicklung, in: Die Tatwelt, Jg. VI, Heft 1/3 (Januar/März), 33-37.

Eucken, W. (1932a): Religion - Wirtschaft - Staat. Zur Problematik des Gegenwartsmenschen, in: Die Tatwelt, Jg. VIII, Heft 2 (Februar), 82-89.

Eucken, W. (1932b): Staatliche Strukturwandlungen und die Krisis des Kapitalismus, in: Weltwirtschaftliches Archiv, Bd. 36, 297-321.

Eucken, W. (1938): Die Überwindung des Historismus, in: Schmollers Jahrbuch, Jg. 62, München.

Eucken, W. (1940): Wissenschaft im Stile Schmollers, in: Weltwirtschaftliches Archiv, Bd. 52/III, 468-506.

Eucken, W. (1948): Das ordnungspolitische Problem, in: Ordo. Jahrbuch für die Ordnung von Wirtschaft und Gesellschaft, Bd. 1, 56-90.

Eucken, W. (1949): Die Wettbewerbsordnung und ihre Verwirklichung, in: Ordo. Jahrbuch für die Ordnung von Wirtschaft und Gesellschaft, Bd. 2, 1-99.

Eucken, W. (1950/1965): Die Grundlagen der Nationalökonomie, Berlin.

Eucken, W. (1952/2004): Grundsätze der Wirtschaftspolitik, Tübingen.

Eucken, W. (2001): Wirtschaftsmacht und Wirtschaftsordnung, Münster.

Goldschmidt, N. (1997): Die Entstehung der Freiburger Kreise, in: Konrad Adenauer Stiftung (Hrsg.): Historisch-Politische Mitteilungen - Archiv für Christlich-Demokratische Politik, Köln, 1-17.

Goldschmidt, N. (2002): Entstehung und Vermächtnis ordoliberalen Denkens, Münster.

Goldschmidt, N. (2003): Theorie auf normativer Basis: Anmerkungen zum ordoliberalen Konzept von Walter Eucken, in: Commun, P. (Hrsg.): L’ordolibéralisme allemande, CergyPontoise, 119-131.

Goldschmidt, N. (Hrsg.) (2005): Wirtschaft, Politik und Freiheit. Freiburger Wirtschaftswissenschaftler und der Widerstand, Tübingen. 
Goldschmidt, N./ Woblgemuth, M. (2008): Entstehung und Vermächtnis der Freiburger Tradition der Ordnungsökonomik, in: dies. (Hrsg.): Grundtexte zur Freiburger Tradition der Ordnungsökonomik, Tübingen, 1-16.

Großmann-Doerth, H. (1933/2008): Selbstgeschaffenes Recht der Wirtschaft und staatliches Recht, in: Goldschmidt, N./ Wohlgemuth, M. (Hrsg.): Grundtexte zur Freiburger Tradition der Ordnungsökonomik, Tübingen, 77-88.

Haselbach, D. (1991): Autoritärer Liberalismus und Soziale Marktwirtschaft, Baden-Baden.

Hayek, F. A. (1970/1996): Die Irrtümer des Konstruktivismus, in: Kerbert, W. (Hrsg.): Die Anmaßung von Wissen, Tübingen, 16-36.

Hayek, F. A. (1971): Die Verfassung der Freiheit, Tübingen.

Hayek, F. A. (1975): Die Anmaßung von Wissen, in: Ordo. Jahrbuch für die Ordnung von Wirtschaft und Gesellschaft, Bd. 26, 12-21.

Homann, K./ Blome-Drees, F. (1992): Wirtschafts- und Unternehmensethik, Göttingen.

Homann, K./ Lütge, C. (2004/2005): Einführung in die Wirtschaftsethik, Münster.

Kant, I. (1977a): Die Metaphysik der Sitten, Frankfurt am M.

Kant, I. (1977b): Schriften zur Anthropologie, Geschichtsphilosophie, Politik und Pädagogik, Frankfurt am M.

Klump, R./ Wörsdörfer, M. (2010): On the Affiliation of Phenomenology and Ordoliberalism: Links between Edmund Husserl, Rudolf and Walter Eucken, in: European Journal of the History of Economic Thought (im Erscheinen).

Leipold, H. (1990): Neoliberal Ordnungstheorie and Constitutional Economics, in: Constitutional Political Economy, Vol. 1, No. 1, 47-65.

Lenel, H. O. (1991): Walter Euckens Briefe an Alexander Rüstow, in: Ordo. Jahrbuch für die Ordnung von Wirtschaft und Gesellschaft, Bd. 42, 11-14.

Miksch, L. (1937/1947): Wettbewerb als Aufgabe, Godesberg.

Nawroth, E. (1961/1962): Die Sozial- und Wirtschaftsphilosophie des Neoliberalismus, Heidelberg.

Nawroth, E. (1965): Zur Sinnerfüllung der Marktwirtschaft, Köln.

Oberender, P./ Christl, C. (2000): Walter Euckens Ordnungstheorie. Eine Vorläuferin der Neuen Institutionenökonomik?, in: Külp, B./ Vanberg, V. (Hrsg.): Freiheit und wettbewerbliche Ordnung, Freiburg, 523-539.

Oswalt, W. (2001): Die falschen Feinde der Offenen Gesellschaft, Vorwort in: Eucken, W.: Wirtschaftsmacht und Wirtschaftsordnung, Münster, 87-152.

Oswalt, W. (2005): Liberale Opposition gegen den NS-Staat, in: Goldschmidt, N. (Hrsg.): Wirtschaft, Politik und Freiheit, Tübingen, 315-353.

Oswalt, W. (2008): Offene Fragen zur Rezeption der Freiburger Schule, in: Goldschmidt, N./ Wohlgemuth, M. (Hrsg.): Grundtexte zur Freiburger Tradition der Ordnungsökonomik, Tübingen, 127-151.

Pies, I. (2002): Theoretische Grundlagen demokratischer Wirtschafts- und Gesellschaftspolitik. Der Beitrag Walter Euckens, in: Pies, I./ Leschke, M. (Hrsg.): Walter Euckens Ordnungspolitik, Tübingen, 1-35.

Ptak, R. (2004): Vom Ordoliberalismus zur Sozialen Marktwirtschaft. Stationen des Neoliberalismus in Deutschland, Opladen.

Ptak, R. (2007): Grundlagen des Neoliberalismus, in: Butterwegge, C./ Lösch, B./ Ptak, R. (Hrsg.): Kritik des Neoliberalismus, Wiesbaden, 13-86. 
Rieter, H./ Schmoly, M. (1993): The Ideas of German Ordoliberalism 1938-1945: Pointing the Way to a New Economic Order, in: European Journal of the History of Economic Thought 1:1, 87-114.

Rüstow, A. (1955): Wirtschaftsethische Probleme der sozialen Marktwirtschaft, in: Boarman, P. (Hrsg.): Der Christ und die soziale Marktwirtschaft, Stuttgart, 53-75.

Schlecht, O. (1990): Zur Ethik in Euckens Werk, in: Bombach, G./ Lenel, H. O./ Schlecht, O. (Hrsg.): Über Walter Euckens „Grundlagen der Nationalökonomie“, Düsseldorf, 63-77.

Schumann, O./ Nutzinger, H. (2009): Ordoliberalismus und Gerechtigkeit: Zum Verhältnis von Eucken und Kant, in: Breuer, M./ Mastronardie, P./ Waxenberger, B. (Hrsg.): Markt, Mensch und Freiheit. Wirtschaftsethik in der Auseinandersetzung, Bern, 55-78.

Suchanek, A. (2001/2007): Ökonomische Ethik, Tübingen.

Ulrich, P. (1994): Integrative Wirtschafts- und Unternehmensethik. Ein Rahmenkonzept, in: Forum für Philosophie (Hrsg.): Markt und Moral. Die Diskussion um die Unternehmensethik, Bern, 75-107.

Ulrich, P. (1997/2008): Integrative Wirtschaftsethik, Bern.

Ulrich, P. (2004): Sich im ethisch-politisch-ökonomischen Denken orientieren. Der St. Galler Ansatz der integrativen Wirtschaftsethik, in: Mieth, D./ Schumann, O./ Ulrich, P. (Hrsg.): Reflexionsfelder integrativer Wirtschaftsethik, Tübingen, 11-28.

Vanberg, V. (2000): Ordnungsökonomik und Ethik, in: Külp, B./ Vanberg, V. (Hrsg.): Freiheit und wettbewerbliche Ordnung, Freiburg, 579-605.

Vanberg, V. (2004): The Freiburg School: Walter Eucken and Ordoliberalism, Freiburg.

Wörsdörfer, M. (2010): On the Economic Ethics of Walter Eucken, in: Konrad Adenauer Stiftung (Hrsg.): 60 Years of Social Market Economy. Formation, Development and Perspectives of a Peacemaking Formula (im Erscheinen). 